\title{
Diel variation in the structure of fish assemblages in south western Amazon streams
}

\author{
Variação diária na estrutura de assembleias de peixes em igarapés da Amazônia Sul \\ Ocidental
}

\author{
Igor David da Costa ${ }^{1 *}$ and Wesclen Vilar Nogueira ${ }^{1}$
}

${ }^{1}$ Laboratório de Ciências Ambientais, Departamento de Engenharia de Pesca, Universidade Federal de Rondônia - UNIR, Rua da Paz, Lino Alves Teixeira, CEP 76196-000, Presidente Médici, RO, Brazil

*e-mail: igordavid@unir.br

Cite as: Costa, I.D. and Nogueira, W.V. Diel variation in the structure of fish assemblages in south western Amazon streams. Acta Limnologica Brasiliensia, 2016, vol. 28, e16.

Abstract: Aim: We investigate the influence of luminosity, habitat conservation and pluviometric periods in fish assemblages of in pasture and forest small streams in western amazon. Methods: Sampling was conducted every two months from July 2013 to April 2014 in nine first- and second-order streams using seine nets and dip nets during the day and night. Fish composition, richness and total abundance were determined for each sampling period. The PERMANOVA was used to evaluate the effects of land use, season, and photoperiod, on fish assemblages. Fish assemblage structure for each stream in the presence and absence of photoperiod was ordered by NMDS analysis. Results: In the light period, 3,484 specimens from 69 species were collected, while 4,574 specimens from 71 species where collected in the dark period. No significant differences in abundance and species richness were recorded between the presence and absence of luminosity periods, rainy and dry seasons and streams in forest and deforested areas. We found evidence of the dark phase composition and richness of exclusive species ( $22 \%$ of species collected were found at night), which were greater than in the light period ( $20 \%$ of species). Conclusion: Despite our failure to identify any nycterohemeral segregation, the results complement existing knowledge of regional ichthyofauna and help provide a better understanding of the distributional, behavioral and functional ecological patterns of fish assemblages.

Keywords: day-night shifts; fish assemblages; Rondônia; Machado River.

Resumo: Objetivo: Investigamos a influência da luminosidade, conservação do habitat e períodos pluviométricos nas assembleias de peixes de igarapés em pastagem e floresta na Amazônia Ocidental. Métodos: A amostragem foi realizada bimestralmente, de julho de 2013 a abril 2014 em nove igarapés de primeira e segunda ordem, utilizando redes de arrasto e puçá durante o dia e a noite. A composição, riqueza e abundância total da ictiofauna foram determinadas para cada período de amostragem. A PERMANOVA foi utilizada para avaliar os efeitos do uso da terra, estação e fotoperíodo sobre assembleias de peixes. A estrutura das assembleias de peixes para cada igarapé com presença e ausência de fotoperíodo foi ordenada através de NMDS. Resultados: No período de presença de luminosidade, 3.484 exemplares de 69 espécies foram coletadas, enquanto 4.574 exemplares de 71 espécies, foram coletados no período com ausência de luminosidade. Não foram encontradas diferenças significativas na abundância e riqueza de espécies entre os períodos de presença e ausência de luminosidade, estação chuvosa e seca e para igarapés em áreas florestadas e desmatadas. Nós encontramos evidências da composição de espécies exclusivas no período com ausência de luminosidade (22\% das espécies), que foi maior do que no período com presença de luminosidade (20\% das espécies). Conclusáo: Apesar 
de nossa incapacidade de identificar qualquer segregação nictemeral, os resultados complementam o conhecimento existente da ictiofauna regional e ajudam a fornecer uma melhor compreensão dos padrôes ecológicos distribucionais, comportamentais e funcionais de assembleias de peixes.

Palavras-chave: substituiçáo diurna-noturna; assembleias de peixes; Rondônia; rio Machado.

\section{Introduction}

Diel activity patterns are among the most evident and detectable rhythms in animals and the main subject of many chronobiological naturalistic and experimental studies (Naruse \& Oishi, 1996; Anras et al., 1997; Volpato \& Trajano, 2006). These patterns have already been investigated in different ecosystems such as seagrass beds, coral reefs and mangroves (Nagelkerken et al., 2000; Pereira et al., 2010). Many organisms studied, from protists to plants and animals, provide strong evidence of a more or less tight control of activity patterns by internal clocks (Volpato \& Trajano, 2006).

Aquatic organisms are not exceptions; many studies have been devoted to different kinds of rhythms these organisms show (Volpato \& Trajano, 2006). In fishes, there is evidence of endogenous and exogenous circadian rhythms in some species, which affects activity patterns, vision and the growth of scales and otoliths (Boujard \& Leatherland, 1992; Volpato \& Trajano, 2006). It has been shown that the teleost circadian system encompasses multiple self-sustained oscillators and that at least two organs, the pineal organ and retina, contain oscillators (Volpato \& Trajano, 2006). Photoreceptors in the retina and pineal organ and deep in the brain are believed to be involved in photosignal transduction to establish circadian rhythms in fish (Iigo \& Tabata, 1996). Each of these organs may be involved individually in the entrainment of locomotor activity in certain fish species (Volpato \& Trajano, 2006).

Diel activity rhythms are expressed by locomotor activity patterns associated with alternating phases (active period $v$ s. rest period), exploitation of habitat, feeding and intra- and interspecific interactions (formation of shoals, agonistic behavior, territory defense, breeding and predator-prey interactions) (Kavaliers, 1980; Helfman, 1986). Taking into account the circadian rhythms, fish can be classified as active during nocturnal, diurnal or crepuscular (mixed types, for which between 35\% and 65\% of activity occurs in the dark phase) periods (Iigo \& Tabata, 1996). For instance, in inland Neotropical waters, Siluriformes and Gymnotiformes are more active at night, whereas most Characiformes and Cichlidae feed and migrate during the day (Lowe-McConnell, 1999; Carvalho, 2008).

Species-specific photoperiod variations can mean that the abundance of individuals and species in a given habitat changes over the course of 24-hours, as determined by feeding activities (Piet \& Guruge, 1997), predation and the need to escape from predators (Gibson et al., 1998; Grossman et al., 1998). According to Lowe-Mcconnell (1999) the presence of predators and large species at dark phase, while small species protect themselves in the vegetation, is a common observation in Neotropical rivers. Many ecological studies of Neotropical fish assemblages have been carried out in larger water bodies, such as rivers and lakes (Arrington \& Winemiller, 2003; Pelicice et al., 2005; Willis et al., 2005; Saccol-Pereira \& Fialho, 2010; Costa \& Freitas, 2010; Duarte et al., 2010, 2012; Costa et al., 2011, 2015).

Despite the immense literature on this topic, little is found regarding fishes - and even less regarding the tropical ones (Volpato \& Trajano, 2006). Especially when related to studies of diurnal and nocturnal assemblages in Amazon small streams (e.g., Bührnheim, 2002). Thus, studies in amazon small streams are necessary, considering the high number of species, the accelerated deforestation and the degradation to the streams, mainly in Rondônia State. It very important acquire the taxonomic, geographical, and ecological knowledge of the ichthyofauna. As a potential additional threat to this fish fauna we can cite the expansion of hydroelectric power plants (Casatti et al., 2013).

Based on the premise that Characiformes is the most abundant and rich group species in amazon small streams, and is more active during the diurnal period, Siluriformes and Gymnotiformes, are most active during the nocturnal period (Lowe McConnell, 1999) and species-specific in the daily cycle variations can mean that the abundance and species in a given habitat changes, determined by intra-interspecific relationships (Gibson et al., 1998; Grossman et al., 1998), we tested the hypothesis that photoperiod influences fish activity, resulting in changes in the composition, abundance and species richness of the icthyofauna in pasture and forest small streams in the rainy and dry seasons, in the Machado River basin, state of Rondônia, Brazil. 


\section{Material and Methods}

\subsection{Study area}

The Machado River is approximately $1,243 \mathrm{~km}$ long. It starts at the confluence of the Pimenta Bueno River and Comemoração River and flows in to the Madeira River in the north of the state of Rondônia (RO) (Fernandes \& Guimarães, 2002). First to third order streams are predominat in the Machado River basin and have a total length of 27,497 km (Krusche et al., 2005). The climate is characterized by temperatures ranging from 19 to $33^{\circ} \mathrm{C}$ and annual rainfall is around $2,500 \mathrm{~mm}$ (Krusche et al., 2005) and there are two well-defined seasons: the dry season (from late May to October) and rainy season (from November to May) (Fernandes \& Guimarães, 2002).

\subsection{Fish collections}

A series of 18 samplings was conducted during three rainy and three dry months: June, August and October 2013 (dry season) and December, February and April 2014 (rainy season). Each sampling included the fish abundance in the nine first- and second-order streams in the middle section of the Machado River basin (Table 1) during high luminosity period (12:00-13:00 hours) and once again during dark period (19:30-20:30 hours).

To prevent the potential negative effects of sampling, samples of the absence luminosity period were conducted $20 \mathrm{~m}$ upstream and 10 days after high luminosity period samplings. We highlight out that both the samples collected during absence luminosity period, as in the high luminosity period, obeyed the minimum distance of $20 \mathrm{~m}$. Failing to collect samples in the same location. Noting that the sites are similar in terms of habitat condition and heterogeneity.
In total, 108 samples were obtained (Table 1 ). Fishes were collected using a seine net $(1.5 \times 2 \mathrm{~m}$, $2 \mathrm{~mm}$ mesh) and dip net $(0.5 \times 0.8 \mathrm{~m}, 2 \mathrm{~mm}$ mesh $)$ along a $50 \mathrm{~m}$ stretch for three people for one hour. Flashlights were used for the nocturnal sampling to find and capture the fishes following Bührnheim (1999).

Fish specimens were euthanized using a lethal dose of clove oil and immediately preserved in a $10 \%$ formalin solution. After two days they were transferred to $70 \%$ alcohol and identified by specialists. Voucher specimens were deposited in the fish collection at the Federal University of Rondônia, Porto Velho, RO, Brazil (Vouchers: UFRO-ICT 023980 to 024000).

In order to better understand the state of those environments, we describe in Table 2 the physical conditions of each sampling site. Environmental variables at each sampling site were characterized following visual observation; substrate composition (i.e., the relative proportion of each substrate component) was visually estimated according to Cummins (1962), and the volume occupied by the submerged vegetation (roots, leaves and stems of the submerged terrestrial vegetation) on each side of the stream was estimated based on the height and width of the vegetation on the banks.

\subsection{Data analysis}

Fish composition, richness $(S)$ and absolute abundance $(\mathrm{N})$ were determined for presence and absence of luminosity periods. The Shapiro-Wilk normality test and Levene's test were applied to determine whether the assumptions used in the parametric (t test) or nonparametric (Mann-Whitney $\mathrm{U}$ test) analyses of abundance $\left(\log _{10}\right)$ and species richness $\left(\log _{10}\right)$ for each photoperiod.

Table 1. Identification of collection site, abbreviation, land use in the adjacent matrix, geographical coordinates and number of samples of the nine streams in the Machado river basin, from July 2013 to May 2014.

\begin{tabular}{|c|c|c|c|c|c|c|c|c|}
\hline \multirow{3}{*}{ Stream } & \multirow{3}{*}{ Abbreviation } & \multirow{3}{*}{ Land use } & \multirow{3}{*}{ Latitude } & \multirow{3}{*}{ Longitude } & \multicolumn{4}{|c|}{ Samples } \\
\hline & & & & & \multicolumn{2}{|c|}{$\begin{array}{l}\text { Luminosity } \\
\text { presence }\end{array}$} & \multicolumn{2}{|c|}{ Luminosity absent } \\
\hline & & & & & Dry & Rainy & Dry & Rainy \\
\hline Penha & PES & Pasture & $11^{\circ} 11^{\prime} 05^{\prime \prime} \mathrm{S}$ & $62^{\circ} 04^{\prime} 40^{\prime \prime} \mathrm{W}$ & 3 & 3 & 3 & 3 \\
\hline Dom João & DJS & Pasture & $11^{\circ} 10^{\prime} 56^{\prime \prime} \mathrm{S}$ & $62^{\circ} 04^{\prime} 51^{\prime \prime} \mathrm{W}$ & 3 & 3 & 3 & 3 \\
\hline Mangueira & MAS & Pasture & $11^{\circ} 13^{\prime} 54^{\prime \prime} \mathrm{S}$ & $62^{\circ} 05^{\prime} 10^{\prime \prime} \mathrm{W}$ & 3 & 3 & 3 & 3 \\
\hline Emerson & SEM & Forested & $11^{\circ} 13^{\prime} 37^{\prime \prime} \mathrm{S}$ & $61^{\circ} 51^{\prime} 16^{\prime \prime} \mathrm{W}$ & 3 & 3 & 3 & 3 \\
\hline 128 & $128 \mathrm{~S}$ & Forested & $11^{\circ} 20^{\prime} 22^{\prime \prime} \mathrm{S}$ & $61^{\circ} 50^{\prime} 23^{\prime \prime} \mathrm{W}$ & 3 & 3 & 3 & 3 \\
\hline Django & DAS & Forested & $11^{\circ} 07^{\prime} 40^{\prime \prime} / \mathrm{S}$ & $61^{\circ} 47^{\prime} 50^{\prime \prime} \mathrm{W}$ & 3 & 3 & 3 & 3 \\
\hline Minuano & MIS & Forested & $11^{\circ} 01^{\prime} 50 " \mathrm{~S}$ & $61^{\circ} 54^{\prime} 42^{\prime \prime} \mathrm{W}$ & 3 & 3 & 3 & 3 \\
\hline Cris & CRS & Pasture & $11^{\circ} 15^{\prime} 31^{\prime \prime} \mathrm{S}$ & $61^{\circ} 51^{\prime} 24^{\prime \prime} \mathrm{W}$ & 3 & 3 & 3 & 3 \\
\hline Douglas & DOS & Pasture & $11^{\circ} 05^{\prime} 08^{\prime \prime} \mathrm{S}$ & $61^{\circ} 53^{\prime} 30^{\prime \prime} \mathrm{W}$ & 3 & 3 & 3 & 3 \\
\hline
\end{tabular}


Table 2. Physiographic descriptors of each stream in the Machado River basin, July 2013 to May 2014.

\begin{tabular}{lccccccccc}
\hline Descriptors & DJS & PES & MAS & 128S & EMS & DAS & MIS & CRS & DOS \\
\hline Macrophytes & $\mathrm{Rs}$ & $\mathrm{Aa}$ & $\mathrm{Re}$ & $\mathrm{X}$ & $\mathrm{X}$ & $\mathrm{X}$ & $\mathrm{Re}$ & $\mathrm{Re}$ & $\mathrm{X}$ \\
Ecotone & $\mathrm{G} / \mathrm{Br}$ & $\mathrm{G}$ & $\mathrm{Aar} / \mathrm{G}$ & $\mathrm{Aar} / \mathrm{Sa}$ & $\mathrm{Aar} / \mathrm{Sa}$ & $\mathrm{Aar} / \mathrm{Sa}$ & $\mathrm{Aar} / \mathrm{Sa}$ & $\mathrm{G}$ & $\mathrm{Aar} / \mathrm{Br}$ \\
Substrate & $\mathrm{S} / \mathrm{Sa} /$ & $\mathrm{A} / \mathrm{R}$ & $\mathrm{Sa} / \mathrm{R} /$ & $\mathrm{Sa} / \mathrm{T} / \mathrm{Gl} /$ & $\mathrm{Sa} / \mathrm{Gl} / \mathrm{R} /$ & $\mathrm{Sa} / \mathrm{T} / \mathrm{Gl} /$ & $\mathrm{Sa} / \mathrm{Tl} /$ & $\mathrm{Sa} / \mathrm{Tl} / \mathrm{Lbt}$ & $\mathrm{Sa} / \mathrm{R}$ \\
& $-\mathrm{Lbt}$ & & $-\mathrm{Lbt}$ & $\mathrm{R} /+\mathrm{Lbt}$ & $+\mathrm{Lbt}$ & $+\mathrm{Lbt}$ & $\mathrm{Gl} / \mathrm{R} /$ & & \\
& & & & & & & $+\mathrm{Lbt}$ & & \\
& & & & & & & &
\end{tabular}

DJS = Dom João stream; PES = Penha stream; MAS = Mangueira stream; 128S = 128 stream; CRS = Cris stream; EMS $=$ Emerson stream; DAS $=$ Django stream; DOS $=$ Douglas stream; and MIS = Minuano stream. Presence of aquatic plants $(\mathrm{Re}=$ rooted emergent; $\mathrm{Rs}=$ rooted submerged; and $\mathrm{Aa}=$ adhered algae), riparian ecotone vegetation (Aar = Arboreal angiosperms; $\mathrm{Sa}=$ shrubby angiosperms; $\mathrm{G}=$ grasses; $\mathrm{Br}=$ bare ravine), substrate $(\mathrm{S}=$ silt; $\mathrm{Sa}=$ sand; $\mathrm{R}=$ rocks; $\mathrm{Tl}=$ thin litter; $\mathrm{Gl}=$ gross litter; and $\mathrm{Lbt}=$ large branches and trunks), $-=$ small amount; + = large amount; and $\mathrm{X}=$ absence.

The Permutational Multivariate Analysis of Variance (PERMANOVA, Anderson, 2001) was used to evaluate the effects of land use (forest and pasture), season (rainy and dry seasons), and photoperiod (presence and absence of luminosity), on biological assemblages, and the interactions between these factors (Anderson, 2001). PERMANOVA is a multi-factorial ANOVA based on any measured distance using permutation methods (Anderson, 2001). The analysis was based on dissimilarity coefficients using Bray-Curtis index (richness and abundance data, were previously Log-transformed) and under 10,000 permutations.

Fish assemblage structure for each stream in the presence and absence of photoperiod was ordered by nonmetric multidimensional scaling (NMDS) analysis, a method for bi- or tridimensional arrangement which represents the association among samples in a similarity matrix (Clarke \& Warwick, 1994). The method is considered robust and suitable for ordination ecological data (Minchin, 1987). To quantify the similarity between sites we used the Bray-Curtis distance measure applied data previously Log-transformed (Clarke \& Gorley, 2001). We performed Mann-Whitney tests with NMDS axis 1 and 2 scores, to detect differences in fish assemblages in the presence and absence of photoperiod.

The PERMANOVA and Mann-Whitney test were performed in the $\mathrm{R}$ environment (R Development Core Team, 2013). The NMDS analyses was carried out using PAST version 2.17 (Hammer et al., 2001). Differences were considered significant when $\mathrm{p} \leq 0.05$.

\section{Results}

A total of 8,058 specimens representing 4 orders, 20 families and 88 species were collected. For both periods, Knodus heteresthes (Eigenmann, 1908) $\left(\mathrm{N}_{\text {light }}=1,425, \mathrm{~N}_{\text {dark }}=2,210\right)$, Serrapinnus notomelas $\left(\right.$ Eigenmann, 1915) $\left(\mathrm{N}_{\text {light }}=357, \mathrm{~N}_{\text {dark }}=413\right)$ and Bryconops giacopinii (Fernández-Yépez, 1950) $\left(\mathrm{N}_{\text {light }}=258, \mathrm{~N}_{\text {dark }}=272\right)$ were the most abundant species. A total of 18 species (20\%) were not found during the diurnal period and $20(22 \%)$ were not found during the nocturnal period (Table 3 ).

Sampling during the presence of luminosity period accounted for 3,484 specimens, 4 orders, 15 families and 69 species. During the absence of luminosity period, 4,574 specimens belonging to 4 orders, 20 families and 71 species were collected. No statistically significant differences were found in abundance $(U=869.0, p=0.90)$ or species richness $(U=867.0, p=0.89)$ between periods of presence and absence of luminosity. Considering all the samples together, Characiformes had the highest abundance and species richness, followed by Siluriformes, Perciformes and Gymnotiformes (Table 4).

The fish assemblages no differed significantly between seasons, type of land use and luminosity, both when considering the abundance and richness of species (Table 5). NMDS analysis of samples based on species abundance did not reveal any separation on axis 1 and 2 retained for interpretation (Stress $=0.41)$. Scores on axis $1(U=820.50$, $p=0.58)$ and axis $2(U=845.00, p=0.74)$ of the NMDS no differed with respect to period for fish assemblages.

\section{Discussion}

According to Lowe-McConnell (1999), the predominant groups in the Amazon basin are Characiformes (43\%), Siluriformes (36\%) and Gymnotiformes (3\%), which account for approximately $82 \%$ of the Amazonian fish assemblage. The predominance of these orders has been reported in several rivers and small streams in the Neotropics (Sabino \& Zuanon, 1998; Lowe-McConnell, 1999; Castro, 1999; 
Table 3. Abundance data for species collected in presence of luminosity (PL) and absence of luminosity (AL) in each stream in the Machado River basin, July 2013 to May 2014.

\begin{tabular}{|c|c|c|c|c|c|c|c|c|c|c|c|c|c|c|c|c|c|c|c|}
\hline \multirow{2}{*}{ Taxa } & \multicolumn{2}{|c|}{$128 S$} & \multicolumn{2}{|c|}{ CRS } & \multicolumn{2}{|c|}{ DAS } & \multicolumn{2}{|c|}{ DJS } & \multicolumn{2}{|c|}{ DOS } & \multicolumn{2}{|c|}{ EMS } & \multicolumn{2}{|c|}{ MAS } & \multicolumn{2}{|c|}{ MIS } & \multicolumn{2}{|c|}{ PES } & \multirow{2}{*}{ Total } \\
\hline & PL & $\mathrm{AL}$ & PL & $\overline{\mathrm{AL}}$ & PL & $\overline{\mathrm{AL}}$ & PL & $\overline{A L}$ & $\overline{\mathrm{PL}}$ & $\overline{A L}$ & $\mathrm{PL}$ & $\overline{\mathrm{AL}}$ & PL & $\overline{\mathrm{AL}}$ & $\overline{P L}$ & $\overline{\mathrm{AL}}$ & $\overline{P L}$ & $\mathrm{AL}$ & \\
\hline
\end{tabular}

\section{Acestrorhynchidae}

Acestrorhynchus

falcatus (Bloch,

1794)

\section{Anostomidae}

Leporinus friderici

(Block, 1794)

Leporinus gomesi

Garavello \& Santos,

1981

\section{Characidae}

Astyanax anterior

Eigenmann, 1908

Astyanax cf.

bimaculatus

(Linnaeus, 1758)

Astyanax

cf. maximus

(Steindachner,

1876)

Astyanax sp.

Brachychalcinus

copei (Steindachner

1882)

Bryconops

giacopinii (Wingert \&

Malabarba, 2011)

Bryconops

piracolina Wingert \&

Malabarba, 2011

Creagrutus anary

Fowler, 1913

Creagrutus beni

Eigenmann, 1911

Creagrutus petilus

Vari \& Harold, 2001

Hyphessobrycon

copelandi Durbin

1908

Jupiaba cf. apenima

Zanata, 1997

$\begin{array}{llllllllllllllllllll}\text { Knodus heteresthes } & 62 & 79 & 5 & 5 & 180 & 239 & 119 & 309 & 1 & 0 & 410 & 571 & 601 & 872 & 1 & 0 & 51 & 9 & 3514\end{array}$

Eigenmann, 1908

Moenkhausia

collettii

(Steindachner,

1882)

Moenkhausia

comma Eigenmann

1908

Moenkhausia

cotinho Eigenmann,

1908

Moenkhausia

cf. pankilopteryx

Bertaco \& Lucinda

2006

DJS = Dom João stream; PES = Penha stream; MAS = Mangueira stream; 128S = 128 stream; CRS = Cris stream;

EMS = Emerson stream; DAS = Django stream; DOS = Douglas stream; and MIS = Minuano stream. 
Table 3. Continued...

\begin{tabular}{|c|c|c|c|c|c|c|c|c|c|c|c|c|c|c|c|c|c|c|c|}
\hline \multirow{2}{*}{ Taxa } & \multicolumn{2}{|c|}{$128 \mathrm{~S}$} & \multicolumn{2}{|c|}{ CRS } & \multicolumn{2}{|c|}{ DAS } & \multicolumn{2}{|c|}{ DJS } & \multicolumn{2}{|c|}{ DOS } & \multicolumn{2}{|c|}{ EMS } & \multicolumn{2}{|c|}{ MAS } & \multicolumn{2}{|c|}{ MIS } & \multicolumn{2}{|c|}{ PES } & \multirow{2}{*}{ Total } \\
\hline & $\mathrm{PL}$ & $\mathrm{AL}$ & $\mathrm{PL}$ & $\mathrm{AL}$ & PL & $\overline{A L}$ & $\mathrm{PL}$ & $\mathrm{AL}$ & $\mathrm{PL}$ & $\overline{A L}$ & $\mathrm{PL}$ & $\overline{A L}$ & PL & $\mathrm{AL}$ & $\mathrm{PL}$ & $\overline{\mathrm{AL}}$ & $\mathrm{PL}$ & $\mathrm{AL}$ & \\
\hline $\begin{array}{l}\text { Moenkhausia } \\
\text { oligolepis (Günther, } \\
\text { 1864) }\end{array}$ & 9 & 28 & 0 & 0 & 41 & 25 & 2 & 6 & 1 & 0 & 4 & 17 & 7 & 14 & 2 & 0 & 4 & 0 & 160 \\
\hline $\begin{array}{l}\text { Moenhhausia aff. } \\
\text { Colletii }\end{array}$ & 0 & 0 & 0 & 0 & 0 & 0 & 0 & 0 & 0 & 0 & 0 & 0 & 33 & 61 & 0 & 0 & 0 & 0 & 94 \\
\hline $\begin{array}{l}\text { Moenkhausia aff. } \\
\text { Cotinho }\end{array}$ & 0 & 0 & 0 & 0 & 0 & 0 & 0 & 2 & 0 & 0 & 0 & 0 & 0 & 0 & 0 & 0 & 0 & 0 & 2 \\
\hline $\begin{array}{l}\text { Moenhhausia aff. } \\
\text { lepidura alta }\end{array}$ & 0 & 0 & 0 & 0 & 0 & 0 & 0 & 0 & 0 & 0 & 0 & 0 & 137 & 75 & 0 & 0 & 0 & 0 & 212 \\
\hline $\begin{array}{l}\text { Phenacogaster beni } \\
\text { Eigenmann, } 1911\end{array}$ & 0 & 2 & 0 & 0 & 0 & 0 & 0 & 0 & 0 & 0 & 0 & 0 & 0 & 0 & 0 & 1 & 0 & 0 & 3 \\
\hline $\begin{array}{l}\text { Phenacogaster } \\
\text { retropinnus Lucena } \\
\text { \& Malabarba, } 2010\end{array}$ & 5 & 1 & 0 & 0 & 0 & 0 & 15 & 63 & 0 & 0 & 0 & 1 & 14 & 9 & 0 & 0 & 0 & 0 & 108 \\
\hline $\begin{array}{l}\text { Poptella compressa } \\
\text { (Günther, 1864) }\end{array}$ & 0 & 0 & 0 & 0 & 0 & 0 & 12 & 9 & 0 & 0 & 0 & 0 & 0 & 0 & 0 & 0 & 0 & 0 & 21 \\
\hline $\begin{array}{l}\text { Serrapinnus } \\
\text { notomelas } \\
\text { (Eigenmann, 1915) }\end{array}$ & 5 & 0 & 0 & 0 & 0 & 0 & 162 & 305 & 2 & 0 & 0 & 0 & 169 & 103 & 0 & 0 & 19 & 5 & 770 \\
\hline $\begin{array}{l}\text { Serrapinnus } \\
\text { microdon } \\
\text { (Eigenmann, 1915) }\end{array}$ & 95 & 9 & 0 & 0 & 3 & 0 & 6 & 29 & 0 & 0 & 1 & 10 & 16 & 17 & 0 & 0 & 1 & 0 & 187 \\
\hline $\begin{array}{l}\text { Tetragonopterus } \\
\text { argenteus Cuvier, } \\
1816\end{array}$ & 0 & 1 & 0 & 0 & 0 & 0 & 0 & 0 & 0 & 0 & 0 & 0 & 0 & 0 & 0 & 0 & 0 & 0 & 1 \\
\hline $\begin{array}{l}\text { Xenurobrycon } \\
\text { polyancistrus } \\
\text { Weitzman, } 1987\end{array}$ & 2 & 5 & 0 & 0 & 0 & 0 & 0 & 0 & 0 & 0 & 0 & 0 & 0 & 0 & 0 & 0 & 0 & 0 & 7 \\
\hline \multicolumn{20}{|l|}{ Crenuchidae } \\
\hline $\begin{array}{l}\text { Characidium aff. } \\
\text { Zebra }\end{array}$ & 0 & 0 & 0 & 0 & 0 & 0 & 0 & 0 & 2 & 0 & 0 & 0 & 0 & 0 & 1 & 0 & 0 & 0 & 3 \\
\hline Characidium sp. & 31 & 7 & 0 & 0 & 0 & 0 & 0 & 0 & 0 & 0 & 2 & 1 & 14 & 10 & 0 & 0 & 0 & 0 & 65 \\
\hline Characidium aff. & 12 & 0 & 0 & 0 & 0 & 0 & 0 & 0 & 0 & 0 & 0 & 0 & 0 & 0 & 0 & 0 & 0 & 0 & 12 \\
\hline
\end{tabular}
etheostoma Cope,

1872

\section{Curimatidae}

$\begin{array}{lllllllllllllllllllll}\text { Steindachnerina } & 0 & 0 & 0 & 0 & 0 & 8 & 0 & 0 & 0 & 0 & 1 & 1 & 5 & 9 & 0 & 0 & 2 & 1 & 27\end{array}$ fasciata (Vari \&

Géry, 1985)

Steindachnerina guentheri

(Eigenmann \&

Eigenmann, 1889)

\section{Erythrinidae}

$\begin{array}{lllllllllllllllllllll}\text { Erythrinus erythrinus } & 0 & 0 & 0 & 0 & 1 & 0 & 0 & 0 & 0 & 0 & 0 & 0 & 0 & 0 & 0 & 0 & 0 & 0 & 1\end{array}$ (Bloch \& Schneider, 1801)

$\begin{array}{lllllllllllllllllllll}\text { Hoplias malabaricus } & 2 & 2 & 0 & 0 & 3 & 1 & 1 & 3 & 0 & 0 & 2 & 2 & 1 & 1 & 0 & 0 & 5 & 3 & 26\end{array}$ (Block, 1794)

\section{Parodontidae}

Parodon sp.

\section{Prochilodontidae}

Prochilodus

nigricans Spix \&

Agassiz, 1829

\section{Serrasalmidae}

Myleus asterias

(Müller \& Troschel, 1844)

DJS = Dom João stream; PES = Penha stream; MAS = Mangueira stream; $128 \mathrm{~S}=128$ stream; CRS = Cris stream;

EMS $=$ Emerson stream; DAS $=$ Django stream; DOS = Douglas stream; and MIS = Minuano stream. 
Table 3. Continued...

\begin{tabular}{|c|c|c|c|c|c|c|c|c|c|c|c|c|c|c|c|c|c|c|c|}
\hline \multirow{2}{*}{ Taxa } & \multicolumn{2}{|c|}{$128 S$} & \multicolumn{2}{|c|}{ CRS } & \multicolumn{2}{|c|}{ DAS } & \multicolumn{2}{|c|}{ DJS } & \multicolumn{2}{|c|}{ DOS } & \multicolumn{2}{|c|}{ EMS } & \multicolumn{2}{|c|}{ MAS } & \multicolumn{2}{|c|}{ MIS } & \multicolumn{2}{|c|}{ PES } & \multirow{2}{*}{ Total } \\
\hline & $\mathrm{PL}$ & $\mathrm{AL}$ & $\mathrm{PL}$ & $\mathrm{AL}$ & $\mathrm{PL}$ & $\mathrm{AL}$ & $\mathrm{PL}$ & $\mathrm{AL}$ & $\overline{\mathrm{PL}}$ & $\mathrm{AL}$ & $\overline{P L}$ & $\overline{A L}$ & PL & $\mathrm{AL}$ & $\mathrm{PL}$ & $\mathrm{AL}$ & $\mathrm{PL}$ & $\mathrm{AL}$ & \\
\hline $\begin{array}{l}\text { Myleus setiger } \\
\text { Müller \& Troschel, } \\
1844\end{array}$ & 0 & 0 & 0 & 0 & 0 & 0 & 7 & 4 & 0 & 0 & 0 & 0 & 0 & 2 & 0 & 0 & 0 & 0 & 13 \\
\hline $\begin{array}{l}\text { Serrasalmus } \\
\text { rhombeus } \\
\text { (Linnaeus, 1766) }\end{array}$ & 0 & 0 & 0 & 0 & 0 & 0 & 0 & 1 & 0 & 0 & 0 & 0 & 0 & 0 & 0 & 0 & 0 & 0 & 1 \\
\hline \multicolumn{20}{|l|}{ GYMNOTIFORMES } \\
\hline \multicolumn{20}{|l|}{ Apteronotidae } \\
\hline $\begin{array}{l}\text { Apteronotus } \\
\text { albifrons (Linnaeus, } \\
1766)\end{array}$ & 0 & 0 & 0 & 0 & 0 & 0 & 1 & 1 & 0 & 0 & 0 & 2 & 0 & 2 & 0 & 0 & 0 & 0 & 6 \\
\hline \multicolumn{20}{|l|}{ Gymnotidae } \\
\hline $\begin{array}{l}\text { Gymnotus carapo } \\
\text { Linnaeus, } 1758\end{array}$ & 0 & 1 & 0 & 0 & 0 & 7 & 39 & 45 & 0 & 0 & 0 & 1 & 0 & 2 & 0 & 0 & 29 & 67 & 191 \\
\hline $\begin{array}{l}\text { Gymnotus curupira } \\
\text { Crampton, Thorsen } \\
\text { \& Albert, } 2005\end{array}$ & 0 & 0 & 0 & 0 & 0 & 0 & 0 & 0 & 0 & 0 & 0 & 1 & 1 & 0 & 0 & 0 & 0 & 0 & 2 \\
\hline \multicolumn{20}{|l|}{ Hypopomidae } \\
\hline $\begin{array}{l}\text { Brachyhypopomus } \\
\text { sp. }\end{array}$ & 0 & 0 & 0 & 0 & 0 & 1 & 0 & 0 & 0 & 0 & 0 & 0 & 0 & 0 & 0 & 0 & 0 & 0 & 1 \\
\hline $\begin{array}{l}\text { Brachyhypopomus } \\
\text { pinnicaudatus } \\
\text { (Hopkins, 1991) }\end{array}$ & 4 & 0 & 0 & 0 & 0 & 0 & 0 & 0 & 0 & 0 & 0 & 0 & 0 & 0 & 0 & 0 & 0 & 0 & 4 \\
\hline \multicolumn{20}{|l|}{ Sternopygidae } \\
\hline $\begin{array}{l}\text { Eigenmannia } \\
\text { trilineata López \& } \\
\text { Castello, } 1966\end{array}$ & 0 & 0 & 0 & 0 & 0 & 0 & 11 & 10 & 0 & 0 & 0 & 2 & 0 & 7 & 0 & 0 & 0 & 2 & 32 \\
\hline $\begin{array}{l}\text { Eigenmannia } \\
\text { limbata (Schreiner } \\
\text { \& Miranda Ribeiro, } \\
\text { 1903) }\end{array}$ & 0 & 0 & 0 & 0 & 0 & 0 & 0 & 0 & 0 & 0 & 0 & 1 & 0 & 0 & 0 & 0 & 1 & 0 & 2 \\
\hline Eigenmannia sp. & 0 & 0 & 0 & 0 & 1 & 0 & 0 & 0 & 0 & 0 & 0 & 1 & 0 & 0 & 0 & 0 & 0 & 0 & 2 \\
\hline $\begin{array}{l}\text { Sternopygus } \\
\text { macrurus (Bloch \& }\end{array}$ & 6 & 1 & 0 & 0 & 0 & 4 & 51 & 53 & 0 & 0 & 0 & 10 & 1 & 3 & 0 & 0 & 0 & 0 & 129 \\
\hline
\end{tabular}

Schneider, 1801)

\section{PERCIFORMES}

\section{Cichlidae}

Aequidens

plagiozonatus

Kullander, 1984

Aequidens rondoni (Miranda Ribeiro,

1918)

Aequidens

tetramerus (Heckel

1840)

Crenicichla lepidota (Heckel 1840)

Crenicichla santosi

Ploeg, 1991

Crenicichla sp.

Satanoperca jurupari (Heckel,

1840)

\section{SILURIFORMES}

\section{Auchenipteridae}

Tatia aulopygia (Kner, 1858)

Tatia sp.

$\begin{array}{lllllllllllllllllll}0 & 0 & 0 & 0 & 0 & 0 & 0 & 0 & 0 & 0 & 0 & 0 & 0 & 0 & 0 & 0 & 2 & 0 & 2 \\ 0 & 0 & 0 & 0 & 27 & 19 & 21 & 12 & 0 & 0 & 2 & 5 & 17 & 29 & 0 & 0 & 53 & 61 & 246 \\ & & & & & & & & & & & & & & & & & & \\ 0 & 0 & 28 & 13 & 0 & 0 & 0 & 0 & 7 & 0 & 0 & 0 & 0 & 0 & 1 & 1 & 0 & 0 & 50 \\ & & & & & & & & & & & & & & & & & & \\ 0 & 0 & 8 & 2 & 0 & 0 & 0 & 0 & 0 & 0 & 0 & 0 & 0 & 0 & 1 & 0 & 0 & 0 & 11 \\ 1 & 3 & 21 & 2 & 8 & 5 & 15 & 4 & 1 & 0 & 1 & 0 & 13 & 6 & 1 & 0 & 17 & 13 & 111 \\ 0 & 0 & 0 & 0 & 0 & 0 & 5 & 2 & 0 & 0 & 7 & 0 & 0 & 0 & 0 & 0 & 3 & 1 & 18 \\ 0 & 0 & 0 & 0 & 0 & 0 & 5 & 12 & 0 & 0 & 0 & 2 & 0 & 0 & 3 & 2 & 0 & 0 & 24\end{array}$

Callichthyidae

DJS = Dom Joáo stream; PES = Penha stream; MAS = Mangueira stream; 128S = 128 stream; CRS = Cris stream; EMS = Emerson stream; DAS = Django stream; DOS = Douglas stream; and MIS = Minuano stream. 
Table 3. Continued...

\begin{tabular}{|c|c|c|c|c|c|c|c|c|c|c|c|c|c|c|c|c|c|c|c|}
\hline \multirow{2}{*}{ Taxa } & \multicolumn{2}{|c|}{$128 S$} & \multicolumn{2}{|c|}{ CRS } & \multicolumn{2}{|c|}{ DAS } & \multicolumn{2}{|c|}{ DJS } & \multicolumn{2}{|c|}{ DOS } & \multicolumn{2}{|c|}{ EMS } & \multicolumn{2}{|c|}{ MAS } & \multicolumn{2}{|c|}{ MIS } & \multicolumn{2}{|c|}{ PES } & \multirow{2}{*}{ Total } \\
\hline & PL & $\overline{\mathrm{AL}}$ & PL & $\overline{\mathrm{AL}}$ & PL & $\overline{\mathrm{AL}}$ & PL & $\mathrm{AL}$ & $\overline{\mathrm{PL}}$ & $\overline{A L}$ & PL & $\overline{\mathrm{AL}}$ & PL & $\mathrm{AL}$ & $\overline{P L}$ & $\overline{\mathrm{AL}}$ & $\overline{P L}$ & $\mathrm{AL}$ & \\
\hline $\begin{array}{l}\text { Corydoras bondi } \\
\text { Gosline, } 1940\end{array}$ & 0 & 11 & 0 & 0 & 0 & 0 & 0 & 0 & 0 & 0 & 0 & 1 & 0 & 0 & 0 & 0 & 0 & 0 & 12 \\
\hline $\begin{array}{l}\text { Corydoras cf. } \\
\text { aeneus (Gill, 1858) }\end{array}$ & 2 & 0 & 0 & 0 & 3 & 0 & 0 & 0 & 0 & 0 & 0 & 0 & 0 & 0 & 0 & 0 & 0 & 0 & 5 \\
\hline $\begin{array}{l}\text { Hoplosternum } \\
\text { littorale (Hancock, } \\
\text { 1828) }\end{array}$ & 0 & 0 & 1 & 0 & 0 & 0 & 0 & 0 & 0 & 0 & 0 & 0 & 0 & 0 & 0 & 0 & 0 & 0 & 1 \\
\hline $\begin{array}{l}\text { Lepthoplosternum } \\
\text { beni Reis, } 1997\end{array}$ & 0 & 0 & 0 & 0 & 0 & 0 & 0 & 0 & 0 & 0 & 0 & 0 & 0 & 1 & 0 & 0 & 1 & 3 & 5 \\
\hline \multicolumn{20}{|l|}{ Heptapteridae } \\
\hline Cetopsorhamdia sp. & 0 & 0 & 0 & 3 & 0 & 0 & 0 & 0 & 0 & 1 & 0 & 0 & 0 & 0 & 0 & 4 & 0 & 0 & 8 \\
\hline $\begin{array}{l}\text { Imparfinis } \\
\text { stictonotus (Fowler, } \\
1940 \text { ) }\end{array}$ & 1 & 2 & 0 & 0 & 0 & 0 & 0 & 0 & 0 & 0 & 0 & 0 & 0 & 0 & 0 & 0 & 0 & 0 & 3 \\
\hline Pimelodella sp. & 0 & 0 & 0 & 0 & 0 & 0 & 0 & 0 & 0 & 1 & 0 & 0 & 0 & 0 & 1 & 2 & 0 & 0 & 4 \\
\hline $\begin{array}{l}\text { Pimelodella howesi } \\
\text { Fowler, } 1940\end{array}$ & 1 & 1 & 0 & 0 & 0 & 0 & 0 & 3 & 4 & 4 & 0 & 0 & 0 & 2 & 1 & 3 & 0 & 0 & 19 \\
\hline $\begin{array}{l}\text { Phenacorhamdia } \\
\text { sp. }\end{array}$ & 0 & 0 & 0 & 0 & 0 & 0 & 0 & 0 & 0 & 0 & 0 & 4 & 0 & 0 & 0 & 0 & 0 & 0 & 4 \\
\hline $\begin{array}{l}\text { Rhamdia quelen } \\
\text { (Quoy \& Gaimard, } \\
\text { 1824) }\end{array}$ & 0 & 0 & 0 & 0 & 3 & 4 & 0 & 0 & 0 & 0 & 0 & 0 & 0 & 2 & 0 & 0 & 0 & 2 & 11 \\
\hline \multicolumn{20}{|l|}{ Loricariidae } \\
\hline $\begin{array}{l}\text { Ancistrus dubius } \\
\text { Eigenmann \& } \\
\text { Eigenmann, } 1889\end{array}$ & 14 & 5 & 0 & 0 & 20 & 22 & 0 & 0 & 0 & 0 & 34 & 42 & 24 & 33 & 2 & 0 & 8 & 3 & 207 \\
\hline Ancistrus sp. & 7 & 0 & 0 & 0 & 1 & 2 & 0 & 4 & 0 & 0 & 3 & 1 & 10 & 9 & 0 & 0 & 0 & 0 & 37 \\
\hline $\begin{array}{l}\text { Farlowella } \\
\text { amazonum } \\
\text { (Günther, 1864) }\end{array}$ & 2 & 0 & 0 & 0 & 0 & 0 & 0 & 0 & 0 & 0 & 0 & 0 & 0 & 0 & 0 & 0 & 0 & 0 & 2 \\
\hline $\begin{array}{l}\text { Farlowella } \\
\text { oxyrryncha (Kner, } \\
\text { 1853) }\end{array}$ & 0 & 0 & 0 & 0 & 0 & 0 & 1 & 2 & 0 & 0 & 1 & 0 & 0 & 2 & 0 & 1 & 0 & 0 & 7 \\
\hline $\begin{array}{l}\text { Hypostomus cf. } \\
\text { plecostomus } \\
\text { (Linnaeus, 1758) }\end{array}$ & 0 & 0 & 0 & 0 & 0 & 0 & 0 & 0 & 0 & 0 & 0 & 0 & 0 & 8 & 0 & 0 & 1 & 1 & 10 \\
\hline $\begin{array}{l}\text { Hypostomus } \\
\text { pantherinus Kner, } \\
1854\end{array}$ & 0 & 0 & 0 & 0 & 0 & 0 & 8 & 6 & 0 & 0 & 0 & 0 & 0 & 0 & 0 & 0 & 0 & 0 & 14 \\
\hline $\begin{array}{l}\text { Hypostomus } \\
\text { pyrineusi (Miranda } \\
\text { Ribeiro, 1920) }\end{array}$ & 2 & 0 & 0 & 0 & 2 & 1 & 1 & 6 & 0 & 0 & 52 & 12 & 1 & 1 & 0 & 0 & 0 & 0 & 78 \\
\hline Hypostomus sp. & 0 & 1 & 0 & 0 & 0 & 0 & 15 & 7 & 0 & 1 & 0 & 0 & 0 & 14 & 0 & 0 & 0 & 0 & 38 \\
\hline $\begin{array}{l}\text { Rineloricaria castroi } \\
\text { Isbrücker \& Nijssen, } \\
1984\end{array}$ & 0 & 0 & 0 & 0 & 0 & 0 & 0 & 0 & 0 & 0 & 0 & 0 & 11 & 0 & 0 & 0 & 0 & 0 & 11 \\
\hline $\begin{array}{l}\text { Rineloricaria } \\
\text { lanceolata (Günther, } \\
\text { 1868) }\end{array}$ & 4 & 7 & 0 & 0 & 1 & 1 & 24 & 30 & 0 & 0 & 2 & 0 & 9 & 31 & 0 & 0 & 0 & 0 & 109 \\
\hline $\begin{array}{l}\text { Rineloricaria } \\
\text { phoxocephala } \\
\text { (Eigenmann \& } \\
\text { Eigenmann, 1889) }\end{array}$ & 0 & 0 & 0 & 0 & 0 & 1 & 0 & 0 & 0 & 0 & 0 & 0 & 2 & 2 & 0 & 0 & 0 & 0 & 5 \\
\hline Rineloricaria sp. & 0 & 3 & 0 & 1 & 0 & 0 & 0 & 0 & 0 & 0 & 0 & 0 & 0 & 1 & 1 & 0 & 0 & 0 & 6 \\
\hline Rineloricaria sp. 1 & 0 & 0 & 0 & 0 & 0 & 0 & 10 & 0 & 0 & 0 & 0 & 0 & 0 & 0 & 0 & 0 & 0 & 0 & 10 \\
\hline $\begin{array}{l}\text { Spatuloricaria } \\
\text { evansii } \\
\text { (Boulenger, 1892) }\end{array}$ & 0 & 0 & 0 & 0 & 0 & 0 & 0 & 0 & 0 & 0 & 3 & 0 & 0 & 0 & 0 & 0 & 0 & 0 & 3 \\
\hline \multicolumn{20}{|l|}{ Pseudopimelodidae } \\
\hline $\begin{array}{l}\text { Batrochoglanis sp. } \\
\text { Trichomycteridae }\end{array}$ & 0 & 0 & 0 & 0 & 0 & 0 & 0 & 1 & 0 & 0 & 0 & 0 & 0 & 0 & 0 & 0 & 0 & 0 & 1 \\
\hline
\end{tabular}

DJS = Dom João stream; PES = Penha stream; MAS = Mangueira stream; 128S = 128 stream; CRS = Cris stream; EMS $=$ Emerson stream; DAS $=$ Django stream; DOS = Douglas stream; and MIS = Minuano stream. 
Table 3. Continued...

\begin{tabular}{|c|c|c|c|c|c|c|c|c|c|c|c|c|c|c|c|c|c|c|c|}
\hline \multirow{2}{*}{ Taxa } & \multicolumn{2}{|c|}{$128 \mathrm{~S}$} & \multicolumn{2}{|c|}{ CRS } & \multicolumn{2}{|c|}{ DAS } & \multicolumn{2}{|c|}{ DJS } & \multicolumn{2}{|c|}{ DOS } & \multicolumn{2}{|c|}{ EMS } & \multicolumn{2}{|c|}{ MAS } & \multicolumn{2}{|c|}{ MIS } & \multicolumn{2}{|c|}{ PES } & \multirow{2}{*}{ Total } \\
\hline & PL & $\overline{A L}$ & PL & $\overline{A L}$ & PL & $\overline{A L}$ & PL & $\overline{A L}$ & PL & $\overline{A L}$ & PL & $\overline{\mathrm{AL}}$ & PL & $\overline{A L}$ & $\mathrm{PL}$ & $\overline{A L}$ & PL & AL & \\
\hline $\begin{array}{l}\text { Ituglanis } \\
\text { amazonicus } \\
\text { (Steindachner, } \\
\text { 1882) }\end{array}$ & 0 & 1 & 0 & 0 & 0 & 0 & 1 & 0 & 0 & 0 & 0 & 0 & 0 & 2 & 0 & 0 & 0 & 0 & 4 \\
\hline Total & 344 & 214 & 66 & 26 & 361 & 396 & 746 & 1213 & 23 & 7 & 604 & 802 & 1184 & 1488 & 19 & 15 & 246 & 309 & \\
\hline
\end{tabular}

DJS = Dom João stream; PES = Penha stream; MAS = Mangueira stream; $128 \mathrm{~S}=128$ stream; CRS = Cris stream;

EMS = Emerson stream; DAS = Django stream; DOS = Douglas stream; and MIS = Minuano stream.

Table 4. Data of numerical abundance $(\mathrm{N})$ and richness $(\mathrm{S})$ for each fish order in periods of presence and absence of luminosity of the nine streams in the Machado river basin, July 2013 to May 2014.

\begin{tabular}{|c|c|c|c|c|c|c|}
\hline \multirow{2}{*}{ Order } & \multicolumn{2}{|c|}{ Presence luminosity } & \multicolumn{2}{|c|}{ Absent luminosity } & \multicolumn{2}{|c|}{ Total } \\
\hline & $\mathbf{N}$ & $\mathrm{s}$ & $\mathbf{N}$ & $\mathrm{s}$ & $\mathbf{N}$ & $\mathbf{s}$ \\
\hline Characiformes & 2,881 & 31 & 3,861 & 38 & 6,742 & 44 \\
\hline Siluriformes & 268 & 23 & 313 & 24 & 581 & 35 \\
\hline Gymnotiformes & 145 & 8 & 219 & 7 & 364 & 9 \\
\hline Perciformes & 189 & 7 & 174 & 6 & 370 & 7 \\
\hline
\end{tabular}

Table 5. PERMANOVA (Permutational Multivariate Analysis of Variance, Pseudo-F value) for fish assemblages in stream in the Machado River basin, July 2013 to May 2014.

\begin{tabular}{|c|c|c|c|c|c|c|c|}
\hline \multirow{2}{*}{ Factors } & \multirow{2}{*}{ d.f } & \multirow{2}{*}{$F$} & \multicolumn{2}{|c|}{ Abundance } & \multirow{2}{*}{$\mathbf{F}$} & \multicolumn{2}{|c|}{ Richness } \\
\hline & & & $\mathbf{R}^{2}$ & $p$ & & $\mathbf{R}^{2}$ & $p$ \\
\hline (1) Season (Rainy and Dry) & 1 & 0.96 & 0.01 & 0.35 & 0.96 & 0.01 & 0.34 \\
\hline (2) Land use (Forest and Pasture) & 1 & 2.29 & 0.02 & 0.09 & 2.29 & 0.02 & 0.10 \\
\hline (3) Luminosity (Presence and Absent) & 1 & 1.41 & 0.01 & 0.22 & 1.41 & 0.01 & 0.22 \\
\hline Interaction (1) × (2) & 1 & 0.31 & 0.00 & 0.77 & 0.31 & 0.00 & 0.76 \\
\hline Interaction (1) × (3) & 1 & 0.24 & 0.00 & 0.84 & 0.24 & 0.00 & 0.83 \\
\hline Interaction (2) × (3) & 1 & 0.41 & 0.00 & 0.67 & 0.41 & 0.00 & 0.68 \\
\hline Interaction (1) × (2) x (3) & 1 & 0.11 & 0.00 & 0.94 & 0.11 & 0.00 & 0.95 \\
\hline
\end{tabular}

d.f. $=$ degrees of freedom; $p=$ significance based on 10,000 randomizations.

Pouilly et al., 2004; Casatti et al., 2013). The most abundant families in the present study were Characidae, Crenuchidae and Loricariidae, a group widely distributed throughout the Amazon basin (Ferreira et al., 1998; Santos et al., 2006).

Our results corroborating the study by Mojica et al. (2014) in Colombian Amazon streams, who found no significant differences in abundance and species richness between light and dark phase. A dual phasing capacity, which is characteristic of a highly adaptable circadian system, appears to be a common fish trait, especially in temperate species, and an adaptation to marked seasonal changes in photoperiod, temperature and food availability (Volpato \& Trajano, 2006). In tropical regions, however, where seasonal changes are not as marked as in temperate zones, this capacity does not appear to be adaptive, corroborating the pattern observed in our study. In tropical areas with well-defined rain cycles, there may be important annual fluctuations in the quantity and quality of food available (Volpato \& Trajano, 2006).

Temporal niche partitioning predicts that different species may be limited by the same resources but differ in terms of when they exploit the resource (Chesson, 1985). Previous studies have shown that the composition of fish populations can vary between light and dark periods (Volpato \& Trajano, 2006). Arrington \& Winemiller (2003) found that Characiformes were a dominant group in both periods, accounting for $80 \%$ and $97 \%$ of abundance during diurnal and nocturnal periods, respectively. This was also observed in the present study, where $82 \%$ and $84 \%$ of the abundance of Characiformes was represented in the diurnal and nocturnal periods, respectively, composed mainly by species $K$. heteresthes, $S$. notomelas and B. giacopinii (Characiformes order) which represented $60 \%$ of total abundance. These species need of high visual acuity, given that these are nektonic species that 
collect food items drifting at mid-water and at the surface, predominantly in the main channel (Ceneviva-Bastos \& Casatti, 2007; Carvalho, 2008; Brejāo et al., 2013; Nogueira \& Costa, 2014). Characiformes collected exclusively during nocturnal samplings belong to species that have large eyes, which presumably increase visual acuity in environments with limited light (Shand, 1997). This anatomical modification is described as an adaptation for foraging in deep water, where diurnal light is limited (Stewart et al., 2002) and capturing food in shallow waters with reduced nocturnal light (Arrington \& Winemiller, 2003).

The variations are related to the different activities that these fishes perform, such as feeding, breeding and moving (Lowe-McConnell, 1999). Characiformes and Perciformes are visually oriented fish with diurnal habits and are found mostly in clear-water environments, while fish guided by chemical, electrical or tactile stimulus, such as Siluriformes and Gymnotiformes, are more active at night and found mostly in turbid waters (Matthews, 1998). Siluriformes from the genera Trichomycterus (Trichomycteridae) and Ancistrus (Loricariidae), which are generally nocturnal (Buck \& Sazima, 1995; Casatti \& Castro, 1998), were reported during the diurnal period, which is uncommon among small loricariids (Volpato \& Trajano, 2006).

The absence of temporal segregation of fish assemblages in our study, corroborates the results of Arrington \& Winemiller (2003). Many fish species have a daily movement pattern from marginal areas to open waters and vice versa and they are often members of different spatially defined assemblages (Matthews, 1998; Arrington \& Winemiller, 2003; Costa et al., 2011). This spatial segregation makes it more difficult to detect nycterohemeral differences when assemblages of fish from specific habitats are analyzed. The lack of segregation between the periods analyzed is accounted for by the high similarity in the fish assemblages in both periods, as a large number of individuals from some taxa (e.g., Characiformes, Characidae and Perciformes) are active during the day and night (Barthem, 1987; Sazima \& Machado 1990; Hahn et al., 1999; Costa et al., 2009; Duarte et al., 2010).

Therefore, for fish assemblages in which most of the species are active during both periods, no significant differences between these periods can be expected. Additionally, Willis et al. (2005) described a low degree of species replacement between diurnal and nocturnal periods (absence of light/dark period variation), especially during the dry season in structurally complex environments. In these environments, fishes make fewer movements toward marginal areas (refuges) at night time (Willis et al., 2005), by the fact that structurally more complex environments show a higher stability of resources intrahabitat, resulting in the greater concentration of resources results in less movement between marginal areas and open waters for foraging during the day (Willis et al., 2005; Pelicice et al., 2005). This is corroborated by the structural conditions of the small streams analyzed in our study, which are composed mainly of rocks, logs, branches, exposed roots and emergent and submerged macrophytes (see Table 2).

The homogeneity of the assemblages in the light and dark periods, can also be related the artifact collection resulting from differences in sampling techniques and data analysis mainly in the case of independent studies (Volpato \& Trajano, 2006). We use methods of active collection, which consists in catching fish with use of instruments that affects the environment by altering the structure of the microhabitat (e.g., submerged litter banks, trunks) (Uieda \& Castro, 1999). The greater fish capture efficiency with active methods results in a higher probability of record low abundant species and cryptobiotic habits (Ribeiro \& Zuanon, 2006), active or inactive (sheltered) in each photoperiod.

Despite our failure to identify any segregation based on photoperiod, we provided evidence of the nocturnal composition and richness of exclusive species $(S=20 ; 22 \%)$ of Machado river. While only a few studies have analyzed the fish assemblages in Amazonian streams in absence/presence of luminosity periods, it is reasonable to suppose that temporal niche partitioning could explain the high fish diversity in these assemblages. Hence, more studies on the temporal segregation of Amazonian fish are needed to understand the distributional, behavioral and functional ecological patterns of these assemblages.

\section{Acknowledgements}

We are grateful to PIBIC/UNIR for providing an undergraduate research project scholarship for WVN, an undergraduate student in the Fisheries Engineering Department. We would also like to thank the Instituto Chico Mendes de Conservaçáo da Biodiversidade for providing sampling permits (no. 311560-1/2011) and the Ichthyology and Fish Laboratory at the Federal University of Rondônia, in particular W. Ohara and J. Lima Filho, for assistance 
with the taxonomic identification. Statistica serial number: AX505B150718FA.

\section{References}

ANDERSON, M.J. A new method for non-parametric multivariate analysis of variance. Austral Ecology, 2001, 26, 32-46. http://dx.doi.org/10.1046/j.14429993.2001.01070.x.

ANRAS, M.L.B., LAGARDERE, J.P. and LAFAYE, J.Y. Diel activity rhythm of seabass tracked in a natural environment: group effects on swimming patterns and amplitudes. Canadian Journal of Fisheries and Aquatic Sciences, 1997, 54(1), 162-168. http://dx.doi. org/10.1139/f96-253.

ARRINGTON, D.A. and WINEMILLER, K.O. Diel changeover in sandbank fish communities in a neotropical floodplain river. Journal of Fish Biology, 2003, 63(1), 442-459. http://dx.doi.org/10.1046/ j.1095-8649.2003.00167.x.

BARTHEM, R.B. Uso de redes de espera no estudo de ritmos circadianos de algumas espécies de peixes nos lagos de várzea do rio Solimóes. Revista Brasileira de Zoologia, 1987, 3(7), 409-422.

BOUJARD, T. and LEATHERLAND, J.F. Circadian rhythms and feeding time in fishes. Environmental Biology of Fishes, 1992, 35(1), 109-131. http://dx.doi. org/10.1007/BF00002186.

BREJÃO, G.L., GERHARD, P. and ZUANON, J. Functional trophic composition of the ichthyofauna of forest streams in eastern Brazilian Amazon. Neotropical Ichthyology, 2013, 11(2), 361-373. http:// dx.doi.org/10.1590/S1679-62252013005000006.

BUCK, S.M.C. and SAZIMA, I. An communities of mailed catfishes (Loricariidae) in southeastern Brazil: Distribution, activity, and feeding. Ichthyological Exploration of Freshwaters, 1995, 6(4), 325-332.

BÜHRNHEIM, C.M. Heterogeneidade de habitats: rasos $\mathrm{x}$ fundos em assembleias de peixes de igarapés de terra firme na Amazônia Central. Revista Brasileira de Zoologia, 2002, 19(3), 889-905. http://dx.doi. org/10.1590/S0101-81752002000300026.

BÜHRNHEIM, M.C. Habitat abundance patterns of fish communities in three Amazonian rain forest streams. In A.L. VAL and V.M.F.A. VAL, eds. Biology of tropical fishes. Manaus: INPA, 1999, pp. 63-74.

CARVALHO, L.N. História natural de peixes de igarapés amazônicos: utilizando a abordagem do Conceito do Rio Contínuo. Manaus: INPA, 2008.

CASATTI, L. and CASTRO, R.M.C. A fish community of the São Francisco River headwaters rifles, southeastern Brazil. Ichthyology Explore Freshwaters, 1998, 9(3), 229-242.

CASATTI, L., PÉREZ-MAYORGA, M.A., CARVALHO, F.R., BREJÁO, G.L. and COSTA, I.D. The stream fish fauna from the rio Machado basin, Rondônia State, Brazil. Check List, 2013, 9(6), 1496-1504. http://dx.doi.org/10.15560/9.6.1496.

CASTRO, R.M.C. Evolução da ictiofauna de riachos sul-americanos: padróes gerais e possíveis processos casuais. In E.P. CARAMASCHI, R. MAZZONI and R. PERES-NETO, eds. Ecologia de peixes de riachos. Rio de Janeiro: UFRJ, 1999, pp. 139-155. Série Oecologia Brasiliensis, vol. 6.

CENEVIVA-BASTOS, M. and CASATTI, L. Oportunismo alimentar de Knodus moenkhausii (Teleostei, Characidae): uma espécie abundante em riachos do noroeste do Estado de São Paulo, Brasil. Iheringia: Série Zoologia, 2007, 97(1), 7-15. http:// dx.doi.org/10.1590/S0073-47212007000100002.

CHESSON, P. Coexistence of competitors in spatially and temporally varying environments: a look at the combined effects of different sorts of variability. Theoretical Population Biology, 1985, 28(1), 263-287. http://dx.doi.org/10.1016/0040-5809(85)90030-9.

CLARKE, K.R. and GORLEY, R.N. PRIMER v5: user manual/tutorial. PRIMER-E. Plymouth: Plymouth Marine Laboratory, 2001.

CLARKE, K.R. and WARWICK, R.M. Change in marine communities: an approach to statistical analysis and interpretation. Plymouth: Plymouth Marine Laboratory, 1994.

COSTA, I.D. and FREITAS, C.E.C. Variação nictemeral na composição e abundância da ictiofauna em um trecho do rio Urucu - Coari/Amazonas/Brasil. Revista Colombiana Ciencia Animal, 2010, 2(2), 355-364.

COSTA, I.D., NOGUEIRA, W.V., ROCHA, V.M., ALBUQUERQUE, P.D.T.F., ALMEIDA PEREIRA, J.M. and RIBEIRO FILHO, R.A.R. Variação nictemeral na assembleia de peixes de um trecho de rio na Amazônia ocidental brasileira. Biotemas, 2015, 28(3), 79-92. http://dx.doi.org/10.5007/21757925.2015v28n3p79.

COSTA, I.D., ROCHA, A.C.P.V., LIMA, M.L.C. and ZUANON, J.A. Composição e abundância de peixes da interface entre as águas abertas e bancos de macrófitas e sua dinâmica nos períodos de crepúsculos matutino e vespertino, no lago Catalão, Amazonas, Brasil. Biotemas, 2011, 24(2), 97-101. http://dx.doi. org/10.5007/2175-7925.2011v24n2p97.

COSTA, S.A.G.L., PERETTI, D., PINTO JÚNIOR, J.P.M., FERNANDES, M.A. and GURGEL JÚNIOR, A.M. Espectro alimentar e variação sazonal da dieta de Plagioscion squamosissimus (Heckel, 1840) (Osteichthyes, Sciaenidae) na lagoa do Piató, Assu, Estado do Rio Grande do Norte, Brasil. Acta Scientiarum, 2009, 31(3), 285-292.

CUMMINS, K.H. An evaluation of some techniques for the collection and analysis of benthic samples with special emphasis on lotic waters. American Midland Naturalist, 1962, 67(2), 477-504. http://dx.doi. org/10.2307/2422722. 
DUARTE, C., RAPP PY-DANIEL, L.H. and DEUS, C.P. Fish assemblages in two sandy beaches in lower Purus river, Amazonas, Brazil. Iheringia: Série Zoologia, 2010., 100(4), 319-328. http://dx.doi. org/10.1590/S0073-47212010000400006.

DUARTE, C., SOUZA, V.S. and NUNES, C.O. Variação nictemeral na composição da ictiofauna no lago Catalão (confluência dos rios Solimóes e Negro). Amazon Science, 2012, 1(1), 18-27.

FERNANDES, L.C. and GUIMARÁES, S.C.P. Atlas geoambiental de Rondônia. Porto Velho: SEDAM, 2002.

FERREIRA, E.J.G., ZUANON, J.A.S. and SANTOS, G.M. Peixes comerciais do Médio Amazonas: região de Santarém, Pará. Brasília: IBAMA, 1998.

GIBSON, R.N., PIHL, L., BURROWS, M.T., MODIN, J., WENNHAGE, H. and NICKELL, L.A. Diel movements of in. Marine Ecology Progress Series, 1998, 165, 145-159. http://dx.doi.org/10.3354/ meps 165145 .

GROSSMAN, G.D., RATAJCZAK JUNIOR, R.E., CRAWFORD, M. and FREEMAN, M.C. Assemblage organization in stream fishes: effects of environmental variation and interspecific interactions. Ecological Monographs, 1998, 68(3), 395-420. http:// dx.doi.org/10.1890/0012-9615(1998)068[0395:AO ISFE] 2.0.CO;2.

HAHN, N.S., LOUREIRO, V.E. and DELARIVA, R.L. Atividade alimentar da curvina Plagioscion squamosissimus (Heckel, 1940) (Perciformes, Scianidae) no rio Paraná. Acta Scientiarum, 1999, 21(1), 309-314. http://dx.doi.org/10.4025/ actascibiolsci.v21i0.4438.

HAMMER, Ø., HARPER, D.A.T. and RYAN, P.D. PAST: Paleontological Statistics Software Package for Education and Data Analysis. Palaeontologia Electronica, 2001, 4(1), 1-9.

HELFMAN, G.S. Fish behaviour by day, night and twilight. In: T. J. PITCHER, ed. The behaviour of teleost fishes. London: Croom Helm, 1986, pp. 366-387.

IIGO, M. and TABATA, M. Circadian rhythms of locomotor activity in the goldfish Carassius auratus. Physiology \& Behavior, 1996, 60(3), 775-781. PMid:8873250. http://dx.doi.org/10.1016/00319384(96)00131-X.

KAVALIERS, M. Circadian locomotor activity rhythms of the burbot, Lota lota: seasonal differences in period length and the effect of pinealectomy. Journal of Comparative Physiology, 1980, 136(3), 215-218. http://dx.doi.org/10.1007/BF00657535.

KRUSCHE, A.V., BALLESTER, M.V.R., VICTORIA, R.L., BERNARDES, M.C., LEITE, N.K., HANADA, L., VICTORIA, D.C., TOLEDO, A.M., OMETTO, J.P., MOREIRA, M.Z., GOMES, B.M., BOLSON, M.A., GOUVEIA NETO,
S., BONELlI, N., DEEGAN, L., NEILl, C., THOMAS, S., AUFDENKAMPE, A.K. and RICHEY, J.E. Efeitos das mudanças do uso da terra na biogeoquímica dos corpos d'água da bacia do rio Ji-Paraná, Rondônia. Acta Amazonica, 2005, 35(2), 197-205. http://dx.doi.org/10.1590/S004459672005000200009 .

LOWE-MCCONNELL, R.H. Estudos ecológicos de comunidade de peixes tropicais. São Paulo: Edusp, 1999.

MATTHEWS, W.J. Patterns in freshwaters fish ecology. Oklahoma: University of Oklahoma Press, 1998. http://dx.doi.org/10.1007/978-1-4615-4066-3.

MINCHIN, P.R. An evaluation of the relative robustness of techniques for ecological ordination. Vegetatio, 1987, 69(1), 89-107. http://dx.doi.org/10.1007/ BF00038690.

MOJICA, J.I., LOBÓN-CERVIA, J. and CASTELLANOS, C. Quantifying fish species richness and abundance in Amazonian streams: assessment of a multiple gear method suitable for Terra firme stream fish communities. Fisheries Management and Ecology, 2014, 21(3), 220-233. http://dx.doi.org/10.1111/fme.12067.

NAGELKERKEN, I., DORENBOSCH, M., VERBERK, W.C., DE LA MORINIERE, C.E. and VAN DER VELDE, G. Day-night shifts of fishes between shallow-water biotopes of a Caribbean bay, with emphasis on the nocturnal feeding of Haemulidae e Lutjanidae. Marine Ecology Progress Series, 2000, 194(1), 55-64. http://dx.doi. org/10.3354/meps194055.

NARUSE, M. and OISHI, T. Annual and daily activity rhythms of loaches in an irrigation creek and ditches around paddy fields. Environmental Biology of Fishes, 1996, 47(1), 93-99. http://dx.doi.org/10.1007/ BF00002382.

NOGUEIRA, V.W. and COSTA, I.D. Aspectos da alimentação de Knodus heteresthes (Characiformes, Characidae) no igarapé do Nove, bacia do rio Machado, Rondônia, Brasil. Biotemas, 2014, 27(3), 97-108. http://dx.doi.org/10.5007/21757925.2014v27n3p97.

PELICICE, F.M., AGOSTINHO, A.A. and THOMAZ, S.M. Fish communities associated with Egeria in a tropical reservoir: investigating the effects of plant biomass and diel period. Acta Oecologica, 2005, 27(1), 9-16. http://dx.doi.org/10.1016/j. actao.2004.08.004.

PEREIRA, P.H.C., FERREIRA, B.P. and REZENDE, S.S.M. Community structure of the ichthyofauna associated with seagrass beds (Halodule wrightii) in Formoso River estuary-Pernambuco, Brazil. Pernambuco: Academia Brasileira de Ciências, 2010, pp. 617-628. 
PIET, G.J. and GURUGE, W.A. Diel variation in feeding and vertical distribution of ten co-occurring fish species: consequences for resource partitioning. Environmental Biology of Fishes, 1997, 50(3), 293 307. http://dx.doi.org/10.1023/A:1007390516552.

POUILLY, M., YUNOKI, T., ROSALES, C. and TORRES, L. Trophic structure of fish communities from Mamoré River floodplain lakes (Bolivia). Ecology Freshwater Fish, 2004, 13(1), 245-257. http:// dx.doi.org/10.1111/j.1600-0633.2004.00055.x.

R DEVELOPMENT CORE TEAM. $R$ : a language and environment for statistical computing [online]. Vienna: R Foundation for Statistical Computing, 2013 [viewed 27 May 2016]. Available from: http:// www.R-project.org

RIBEIRO, O.M. and ZUANON, J. Comparação da eficiência de dois métodos de coleta de peixes em igarapés de terra firme da Amazônia Central. Acta Amazonica, 2006, 36(3), 389-394. http://dx.doi. org/10.1590/S0044-59672006000300017.

SABINO, J. and ZUANON, J.A.S. A stream fish communities in Central Amazonia: distribution, activity patterns and feeding behavior. Ichthyological Exploration of Freshwaters, 1998, 8(3), 201-210.

SACCOL-PEREIRA, A. and FIALHO, C.B. Seasonal and diel variation in the fish assemblage of a Neotropical delta in southern Brazil. Iheringia: Série Zoologia, 2010., 100(2), 169-178. http://dx.doi. org/10.1590/S0073-47212010000200013.

SANTOS, G.M., FERREIRA, E. and ZUANON, J. Peixes comerciais de Manaus. Manaus: IBAMA, 2006.
SAZIMA, I. and MACHADO, F.A. Underwater observations of piranhas in western Brazil. Environmental Biology of Fishes, 1990, 28(4), 17-31. http://dx.doi.org/10.1007/BF00751026.

SHAND, J. Ontogenetic changes in retinal structure and visual acuity: A comparative study of coral-reef teleosts with differing post-settlement lifestyles. Environmental Biology of Fishes, 1997, 49(3), $307-$ 322. http://dx.doi.org/10.1023/A:1007353003066.

STEWART, D.J., IBARRA, M. and BARRIGASALAZAR, R. Comparison of deep-river and adjacent sandy-beach fish communities in the Napo River Basin, Eastern Ecuador. Copeia, 2002, 2(2), 333-343.

UIEDA, V.S. and CASTRO, R.M.C. Coleta e fixação de peixes de riachos. In E.P. CARAMASCHI, R. MAZZONI and P.R. PERES-NETO, eds. Ecologia de peixes de riachos. Rio de Janeiro: UFRJ, 1999, pp. 1-22. Série Oecologia Brasiliensis.

VOLPATO, G.L. and TRAJANO, E. Biological rhythms. In L.A. VAL, V.M.F.A. VAL and D.J. RANDAL, eds. Fish physiology. San Diego: Elsevier, 2006, pp. 101-153.

WILLIS, S.C., WINEMILLER, K.O. and LOPEZFERNANDEZ, H. Habitat structural complexity and morphological diversity of fish assemblages in a Neotropical floodplain river. Oecologia, 2005, 142(2), 284-295. PMid:15655689. http://dx.doi. org/10.1007/s00442-004-1723-z.

Received: 27 May 2016 Accepted: 23 September 2016 